\title{
ARTICLE
}

Received 10 Aug 2014 | Accepted 18 Nov 2014 | Published 19 Dec 2014 DOl: 10.1038/ncomms6888

\section{Co-option of alternate sperm activation programs in the evolution of self-fertile nematodes}

Qing Wei ${ }^{1}$, Yanmei Zhao ${ }^{2,3}$, Yiqing Guo ${ }^{2,4}$, Julie Stomel ${ }^{1}$, Ryan Stires ${ }^{1}$ \& Ronald E. Ellis 1,2

Self-fertility evolved independently in three species of Caenorhabditis, yet the underlying genetic changes remain unclear. This transition required that $X X$ animals acquire the ability to produce sperm and then signal those sperm to activate and fertilise oocytes. Here, we show that all genes that regulate sperm activation in C. elegans are conserved throughout the genus, even in male/female species. By using gene editing, we show that $C$. elegans and C. briggsae hermaphrodites use the SPE-8 tyrosine kinase pathway to activate sperm, whereas C. tropicalis hermaphrodites use a TRY-5 serine protease pathway. Finally, our analysis of double mutants shows that these pathways were redundant in ancestral males. Thus, newly evolving hermaphrodites became self-fertile by co-opting either of the two redundant male programs. The existence of these alternatives helps explain the frequent origin of self-fertility in nematode lineages. This work also demonstrates that the new genome-editing techniques allow unprecedented power and precision in evolutionary studies.

\footnotetext{
${ }^{1}$ Graduate School of Biomedical Sciences, Rowan University SOM, B303 Science Center, 2 Medical Center Drive, Stratford, New Jersey 08084, USA.

${ }^{2}$ Department of Molecular Biology, Rowan University SOM, B303 Science Center, 2 Medical Center Drive, Stratford, New Jersey 08084, USA. ${ }^{3}$ Laboratory of Noncoding RNA, Institute of Biophysics, Chinese Academy of Sciences, Beijing 100101, China. ${ }^{4}$ Syracuse University, 107 College Place, Syracuse, New York 13244, USA. Correspondence and requests for materials should be addressed to R.E.E. (email: ellisre@rowan.edu).
} 
$\mathrm{O}$ ne of the major questions in evolutionary biology is how innovation occurs. Darwin ${ }^{1}$ suggested that novel traits could be produced if older features were co-opted for new uses and subsequent research has confirmed this idea ${ }^{2}$. However, neither the qualities that determine which traits are co-opted to produce new features, nor the mechanisms that implement these changes are clear. For example, the genetic changes that led treehoppers to express a modified wing structure on the first thoracic segment ${ }^{3}$ or beetles to produce a head horn ${ }^{4}$ remain obscure.

The origin of self-fertility in nematodes is ideal for tackling the problem ${ }^{5}$. Self-fertile hermaphrodites originated independently in three species of Caenorhabditis nematodes ${ }^{6-8}$ (Fig. 1a). This step required $X X$ animals to acquire the ability to (1) produce sperm and (2) signal those sperm to activate and fertilise oocytes 9 . As these transitions occurred recently, it might be possible to identify the underlying changes by comparative analysis.

Studies with $C$. elegans showed that five genes are required to initiate hermaphrodite sperm activation in that species-spe-8 (refs 10,11), spe-12 (refs 10,12), spe-19 (ref. 13), spe-27 (ref. 14) and spe-29 (ref. 15). Mutations in any of these genes block hermaphrodite self-fertility, but do not prevent male sperm from activating and fertilising oocytes. All five genes are expressed in sperm and three encode transmembrane proteins ${ }^{16}$. Furthermore, SPE- 8 is a protein tyrosine kinase whose localisation to the plasma membrane in spermatids requires the other genes ${ }^{11}$. Thus, these five proteins might define a complex located on the plasma membrane that responds to an extracellular signal controlling activation. Although one candidate for this signal is extracellular $\operatorname{zinc}^{17}$, none of the proteins from the SPE-8 group has known zinc-binding domains or zinc importation motifs.

Recently, the TRY-5 protease was shown to act in a second sperm activation pathway, which functions only in males ${ }^{18,19}$. Before mating, TRY-5 activity is inhibited by SWM-1, a protein with two trypsin inhibitor-like domains ${ }^{20}$. During ejaculation, large TRY-5 reserves are secreted into the seminal fluid, where they activate male spermatids. Although their direct target is unknown, one possibility is SNF-10, a membrane protein found in sperm ${ }^{21}$.

Although try-5 males are fertile, the spe-8; try-5 double mutants are sterile, which indicates that these two signal transduction pathways are redundant in $C$. elegans ${ }^{18}$. However, special mutations in spe-6 (ref. 22), spe-4 (ref. 23) and spe-46 (ref. 24), which are all required for spermatogenesis, can bypass the need for these signals in the activation process. For example, spe-6(hc163) is epistatic to both try-5 (ref. 18) and spe-8 (ref. 22), so the SPE-6 kinase probably acts within spermatids to respond to extracellular signals and cause activation.

Intra-species crosses showed that some aspects of sperm activation are conserved in Caenorhabditis, although the molecular details remain unknown ${ }^{25}$. As experimental studies using $C$. remanei revealed that female nematodes lack a mechanism to activate sperm ${ }^{9}$, learning which methods of sperm activation are used by hermaphrodites from diverse species is critical for understanding the origins of self-fertility. Here, we show that two redundant sperm activation pathways have been conserved in Caenorhabditis. Hermaphrodites from C. elegans and C. briggsae require the SPE- 8 pathway to activate
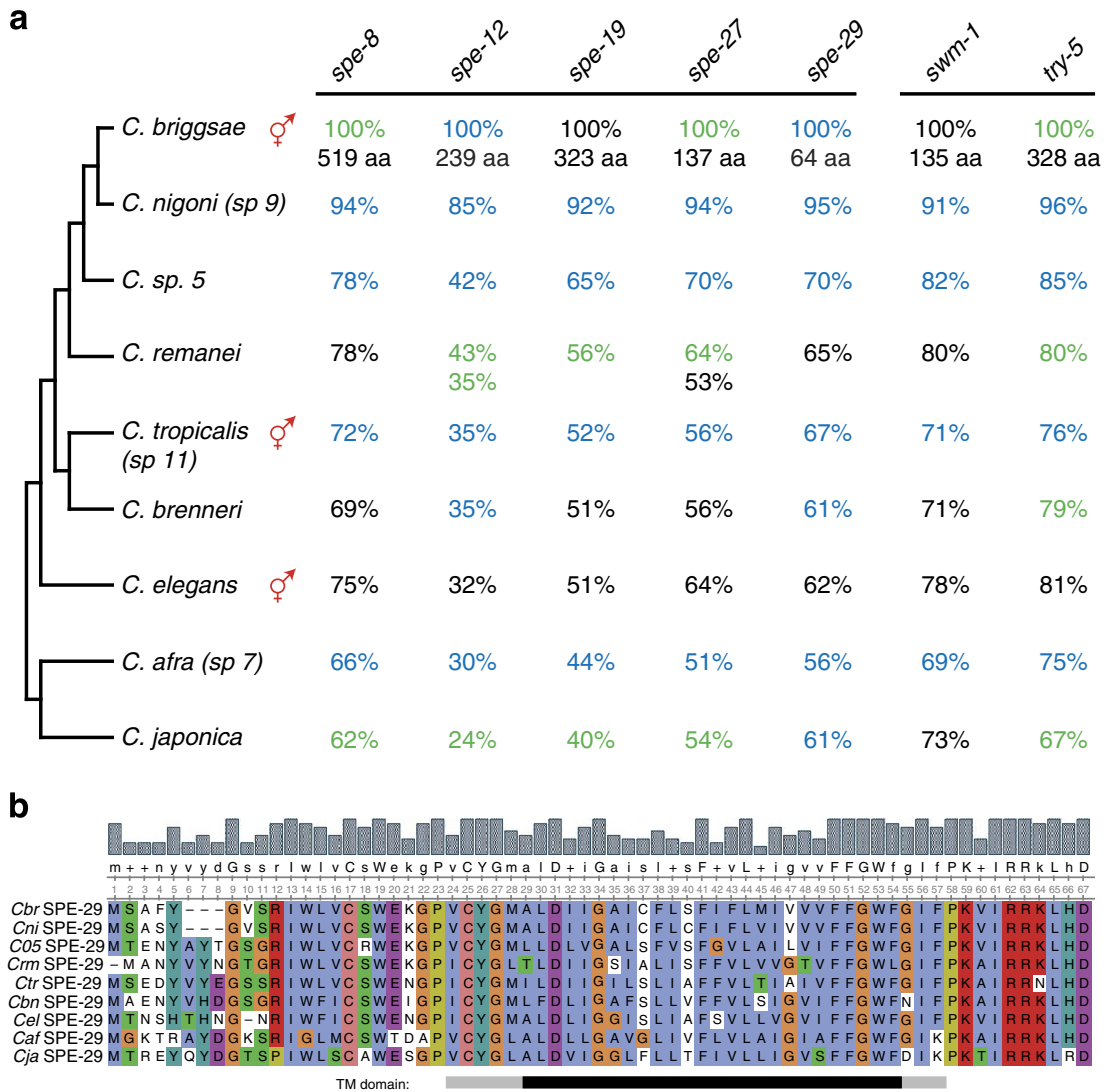

Figure 1 | All sperm activation genes are conserved throughout Caenorhabditis. (a) Percent identity of each protein to its $C$. briggsae ortholog. In the phylogeny ${ }^{8}$ at left, each androdioecious species is marked in red. Percentages for newly described proteins are blue and for revised proteins are green. C. remanei has duplicates of spe- 12 and spe-27. (b) Representative alignment of SPE-29 sequences, prepared using MUSCLE ${ }^{45}$. A conserved transmembrane domain is marked with the core in black and variable edges in grey ${ }^{46}$. For brevity, C. sp. 5 has been abbreviated C05 in the figure. 
their sperm, whereas hermaphrodites from $C$. tropicalis require the TRY-5 pathway. The ability to co-opt either of two alternative signal transduction pathways could explain the frequent origin of self-fertility in this group of animals.

\section{Results}

Sperm activation genes are conserved in Caenorhabditis. To begin, we identified orthologs of all C. elegans sperm activation genes in the eight-related species for which partial genome sequences were available. Previously, only 23 of these sperm activation genes had been identified. Using BLAST searches and phylogenetic comparisons, we refined these predictions and identified the remaining 33 genes (Fig. 1, Supplementary Fig. 1). We used degenerate oligonucleotides to clone $\mathrm{Cbr}$-spe-12, which was missing from the genome assembly, and the PCR to isolate missing parts of Ctr-spe-8 and Ctr-spe-27. Our results show that the ancestor of Caenorhabditis had orthologs of all seven sperm activation genes. Moreover, each of the encoded proteins has conserved elements, like the transmembrane domain of SPE-29 (Fig. 1b) or the kinase domain of SPE-8. As the common ancestor and six species are male/female, none of these genes is likely to function exclusively in hermaphrodites.

We used reverse transcription PCR (RT-PCR) to characterise the expression of each gene in C. briggsae. The five members of the C. elegans spe- 8 group are expressed in spermatids ${ }^{16}$, where they respond to an unknown signal. In $C$. briggsae, their expression is also correlated with spermatogenesis-the transcripts are present in larval hermaphrodites, larval males and adult males, but absent from adult hermaphrodites (Fig. 2a and Supplementary Fig. 2). Thus, genes of the spe-8 group appear to produce sperm proteins in C. briggsae, as they do in C. elegans. By contrast, C. briggsae try-5 and swm-1 transcripts were detected in all animals, including adult hermaphrodites.

The SPE-8 group regulates $X X$ sperm activation in C. briggsae. To learn how these genes functioned, we used TALENs to generate knockout mutants of key members of the spe- 8 group in C. briggsae ${ }^{26-28}$ (Fig. 3a). We found that mutations in Cbr-spe-8 and Cbr-spe-19 caused $X X$ animals to produce inactive spermatids (Fig. 3b), which prevented self-fertility (Fig. 3c). This effect was strongest for frame-shifting alleles, which are probably null, and more mild for Cbr-spe-19(v173), which inserts six amino acids. Because the C. briggsae mutant males were fertile, all of these strains could be maintained as male/hermaphrodite populations, so the mutant oocytes function normally. In addition, some sperm in these mutant hermaphrodites could be transactivated by seminal fluid from males, restoring self-fertility (Fig. 3d). This entire suite of phenotypes resembles that of their $C$. elegans counterparts ${ }^{10,13}$.

As the spe-8 group of genes predated the origin of self-fertility, we wondered if the similarity between C. elegans and C. briggsae was caused by chance or a developmental bias ${ }^{29}$ that favoured the use of these genes in hermaphrodites. Thus, we examined a third androdioecious species-C. tropicalis (formerly sp. 11) (refs 8,30). Null alleles in Ctr-spe-19 and Ctr-spe-27 had only a mild effect on reproduction, and the mutant hermaphrodites were all self-fertile (Fig. 3e). Thus, the spe-8 group is required for hermaphrodite sperm activation in C. elegans and C. briggsae, but not in C. tropicalis. Another pathway must activate sperm in hermaphrodites of that species.

TRY-5 regulates $X X$ sperm activation in C. tropicalis. Next, we studied the try-5 pathway, which regulates sperm activation in C. elegans ${ }^{18}$ and A. suum ${ }^{19}$ males. TRY-5 is a trypsin protease that cleaves an unknown target on the surface of sperm. In

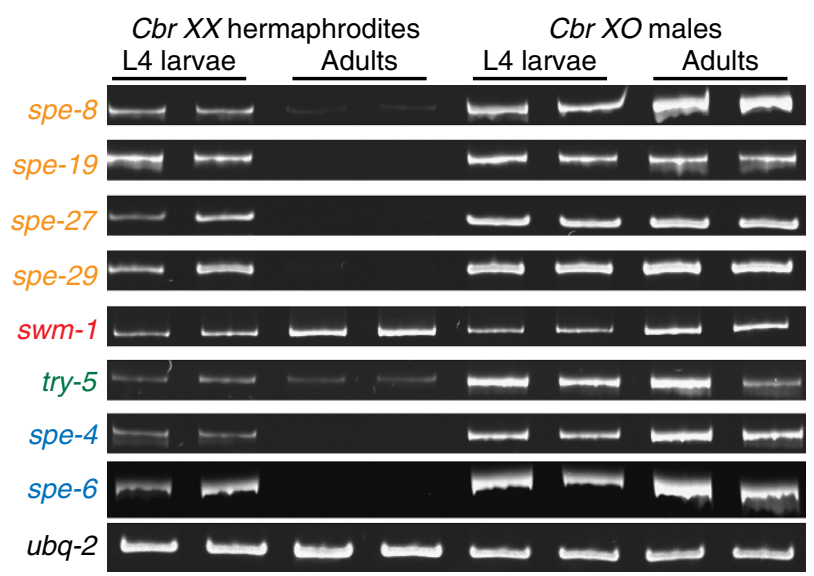

b

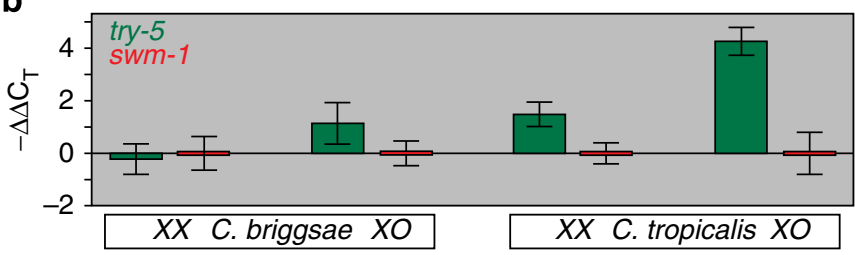

Figure 2 | Sperm activation genes are expressed in males and some hermaphrodites. (a) Semi-quantitative RT-PCR analysis of RNA from independent samples of each indicated age and sex. The genes spe- 4 and spe- 6 were control spermatogenesis genes and the ubiquitin gene $u b q-2$ was a loading control. (b) Real-time RT-PCR analysis of the indicated genes and animals at the L4 larval stage. There were three biological replicates of each sample, each of which had two technical replicates; the error bars represent standard error of the mean. Note that try-5 levels are higher relative to swm- 1 levels in the three groups that use TRY-5 to activate sperm, but not in C. briggsae hermaphrodites, which instead use the spe- 8 pathway.

C. elegans, TRY-5 is secreted by the male gonad, and its activity is blocked by the protease inhibitor SWM-1, preventing premature activation $^{20}$. We found that knocking out $s w m-1$ caused male sperm to activate prematurely in both C. briggsae (Fig. 4a,b) and C. tropicalis (Fig. 5a). These results show that the role of SWM-1 has been conserved. Null alleles of C. briggsae try-5 had no phenotype on their own, but did suppress swm-1 mutations (Fig. 4a,b), implying that Cbr-SWM-1 works through TRY-5 to control activation (Fig. 4c). Similarly, knocking down try-5 activity in C. tropicalis suppressed the premature activation caused by swm-1 mutations in that species (Fig. 5a,b). These phenotypes all resemble those seen in C. elegans ${ }^{8,20}$. However, we were surprised to find that $C t r-t r y-5$ males were infertile, so the spe-8 group of genes is not sufficient for sperm activation in males of this species (Fig. 5c).

When we examined hermaphrodites, we found that C. tropicalis try-5 mutations blocked self-fertility, whereas C. briggsae try-5 mutations had no effect (Fig. 4d). Sterile Ctr-try-5 hermaphrodites produced normal oocytes, because they made cross progeny when fertilised by males. However, some sperm were completely inactive and others had spiky projections rather than pseudopods, which might indicate defective activation (Fig. 4e). Thus, TRY-5 is required for sperm activation in C. tropicalis hermaphrodites, whereas the spe-8 group is essential in C. elegans and C. briggsae hermaphrodites. As one might expect given this result, try-5 expression in C. tropicalis hermaphrodites is high relative to that of $s w m-1$ (Fig. 2b). 
a

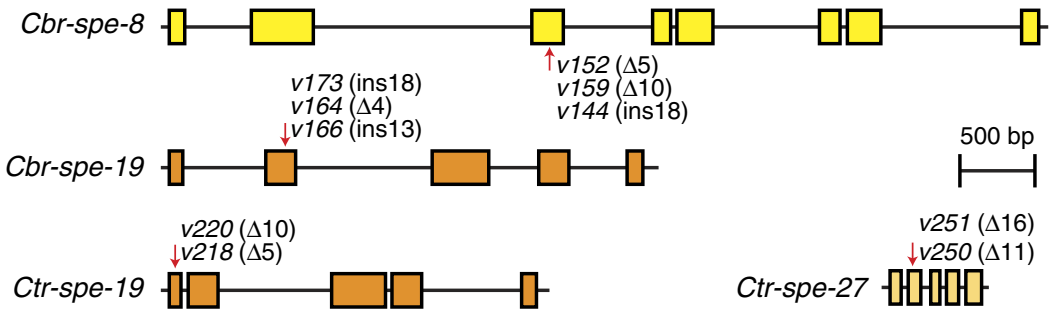

b

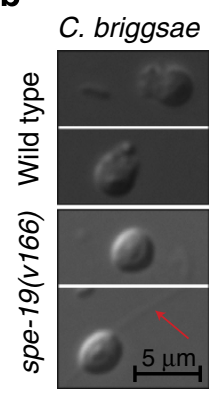

C

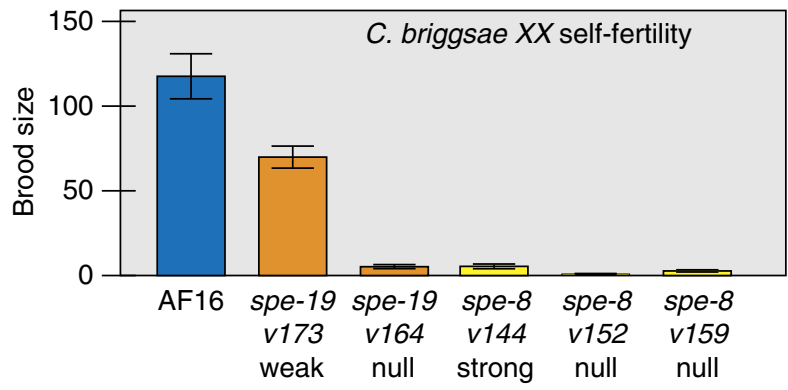

d

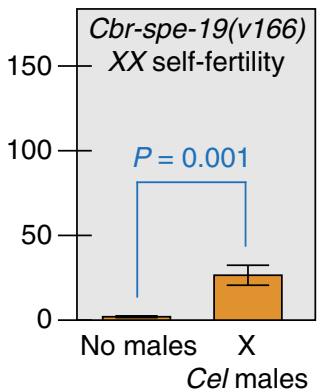

e

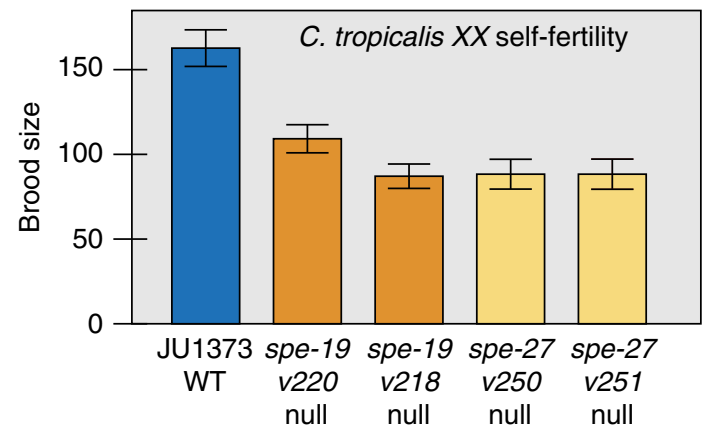

Figure 3 | The spe-8 group controls hermaphrodite sperm activation in C. briggsae but not C. tropicalis. (a) Gene structures. Each $5^{\prime}$ end is left and exons are boxed. Mutation sites are indicated with arrows. (b) Sperm isolated from C. briggsae wild-type hermaphrodites were active, and those from spe-19(v166) were inactive or had only spiky protrusions (red arrow). (c) The spe-8 group is required for sperm activation in C. briggsae hermaphrodites. Brood size is the number of eggs laid by unmated hermaphrodites of each genotype. Bars show standard error of the mean. (d) C. briggsae spe-19 sperm can be transactivated by male seminal fluid. We used C. elegans fog- 2 males, which cannot fertilise C. briggsae ${ }^{47}$. The significance was determined with a Student's $t$-test. (e) The spe-8 group is not required for hermaphrodite sperm activation in $C$. tropicalis.

SPE-8 and TRY-5 pathways were redundant in ancestral males. To learn how these differences originated, we studied C. briggsae males. These experiments were motivated by genetic tests showing that the TRY-5 and SPE- 8 pathways are redundant in C. elegans males ${ }^{18}$. We found that Cbr-try-5 males produced numerous progeny when crossed with Cbr-try-5 spe-19 hermaphrodites, but that Cbr-try-5 spe-19 males produced few or none (Fig. 6a). This decrease in male fertility shows that these sperm activation pathways are redundant in C. briggsae. Given the phylogeny, this result implies that the common ancestor was a male/female species that used both pathways to activate male sperm (Fig. 6b).

Why have these redundant pathways been conserved? Studies of $C$. elegans showed that some mutants in the spe-8 group decreased male fertility, so these genes might control additional aspects of sperm function ${ }^{12,15}$. When we tested Cbr-spe-19 and Ctr-spe-19 males by crossing them with hermaphrodites, we found that their sperm activated but competed poorly for fertilisation (Fig. 7 and Supplementary Fig. 3). Thus, these redundant activation pathways may have persisted because they have additional, non-overlapping functions. However, in C. tropicalis, the overlap between these activation pathways is minimal, as the ctr-try-5 males are sterile (Fig. 5c). Thus, it is possible that the functions of the SPE- 8 and TRY- 5 systems are diverging in this species.

The sister species to $C$. briggsae lacks $X X$ sperm activation. Our data suggested that $X X$ animals co-opt one of the male sperm activation systems during the evolution of self-fertility. However, this model rests on the idea that females in gonochoristic species do not use sperm activations signals, which previously had been tested only in C. remanei ${ }^{9}$. Thus, we carried out similar experiments with the gonochoristic species C. nigoni, which is so closely related to $C$. briggsae that the two can mate and produce fertile offspring 8,31 . We found that $C$. nigoni $X X$ animals that were induced to produce spermatids, as well as oocytes by RNA interference (RNAi) were not self-fertile (Fig. 8a), just as observed with C. remanei. However, when we induced C. nigoni/ C. briggsae hybrids to produce spermatids, their sperm activated and the animals made self-progeny (Fig. 8c). Thus, C. briggsae produces a dominant factor that activates sperm in $X X$ animals, but $C$. nigoni lacks this factor. 
a

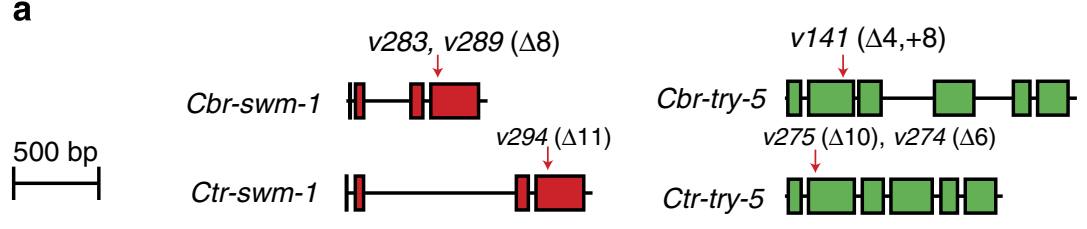

b

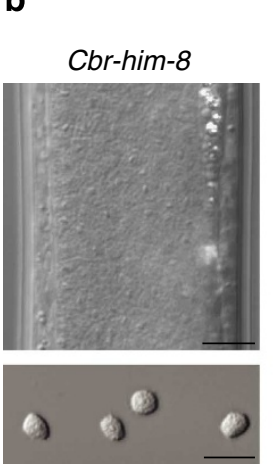

Inactive

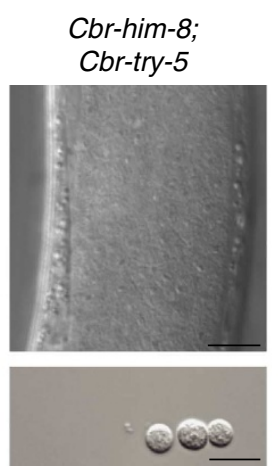

Inactive
Cbr-him-8; Cbr-swm-1

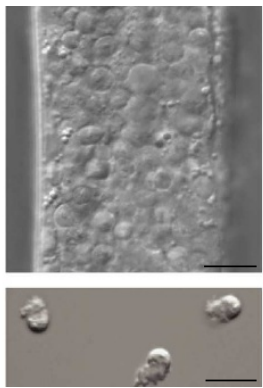

Active
Cbr-him-8; Cbr-swm-1 Cbr-try-5

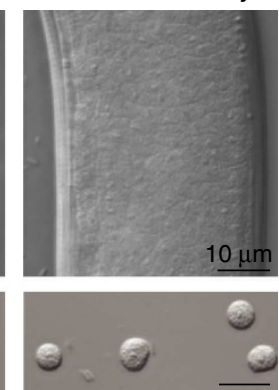

Inactive

c

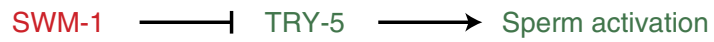

d

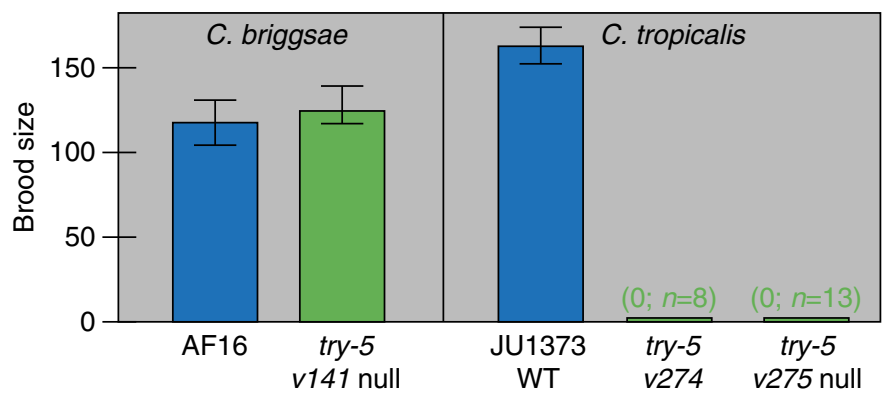

e

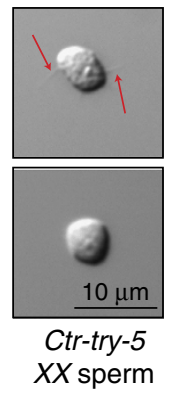

Figure 4 | TRY-5 controls sperm activation in males and C. tropicalis hermaphrodites. (a) Gene structures. Each $5^{\prime}$ end is left and exons are boxed. Mutation sites are indicated with arrows. (b) Differential interference contrast photomicrogaphs of gonads from C. briggsae males 1 day after the L4 larval stage (above) and sperm from dissected males (below). Scale bars, $10 \mu \mathrm{m}$. (c) Summary of the results of genetic epistasis. The swm-1 gene must act through try-5 to regulate male sperm activation, as it does in C. elegans ${ }^{18,20}$. (d) TRY-5 is absolutely required for self-fertility in C. tropicalis hermaphrodites, but not in C. briggsae ones. (e) Spermatids isolated from Ctr-try-5(v275) hermaphrodites have not been activated, although some have spiky projections (red arrows).

\section{Discussion}

These results point to a simple model for the origin of self-fertility in hermaphroditic nematodes. The ancestor of the elegans group was a male/female species ${ }^{6-8}$, and the males used two redundant signals to activate sperm (Fig. 9a), whereas the females did not produce either signal ${ }^{9}$ (Fig. 9b). When C. elegans, C. tropicalis and C. briggsae began the transformation to self-fertility, the $X X$ animals co-opted one or the other of the male pathways (Fig. 9c,d), but did not need both. As predicted by this model, C. briggsae contains a dominant factor that can activate sperm in intra-species hybrids.

The ability to co-opt specific genetic programs might underlie the origin of many complex traits ${ }^{32}$ and could explain some examples of parallel evolution ${ }^{33}$. Our data strongly support this model, as we show that nematodes have co-opted male sperm activation programs for use in newly evolving hermaphrodites. More importantly, we found that two different sperm activation programs can be co-opted for this purpose. Usually, developmental biases or constraints are thought to prevent certain types of variation from occurring, which prevents some evolutionary transitions ${ }^{29}$. Our results imply that some patterns of developmental regulation favour certain types of change, as the existence of two sperm activation pathways in nematodes helps explain why self-fertility has originated so frequently in this group $^{8}$. Thus, this system provides a concrete example of the concept of evolvability in evolutionary developmental biology ${ }^{34}$.

Although cases of convergent or parallel evolution provide natural experiments that illuminate evolutionary processes, their definitions remain controversial ${ }^{35,36}$. Usually, they are thought of as distinct phenomena-parallel evolution occurs through the independent origin and fixation of mutations affecting the same gene, whereas convergence occurs when selection leads to different solutions to the same problem. Our results show that this distinction is often artificial, as the two Caenorhabditis species evolved self-fertility by recruiting the spe- 8 pathway to control hermaphrodite sperm activation (parallel evolution) but a third achieved the same end by recruiting the try-5 pathway (convergent evolution).

Finally, our work shows that entire pathways in newly described species can be subjected to precise evolutionary genetic 
a

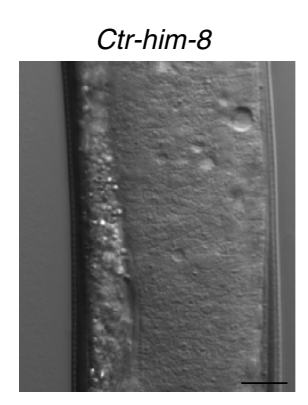

Ctr-him-8; swm-1

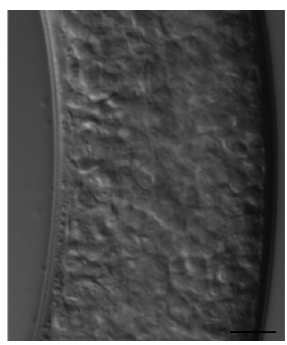

Ctr-him-8; swm-1 try-5(RNAi)

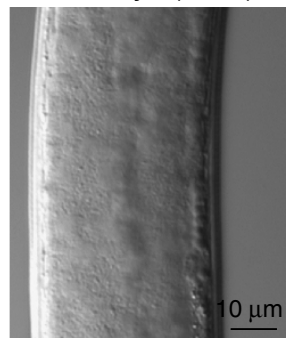

b

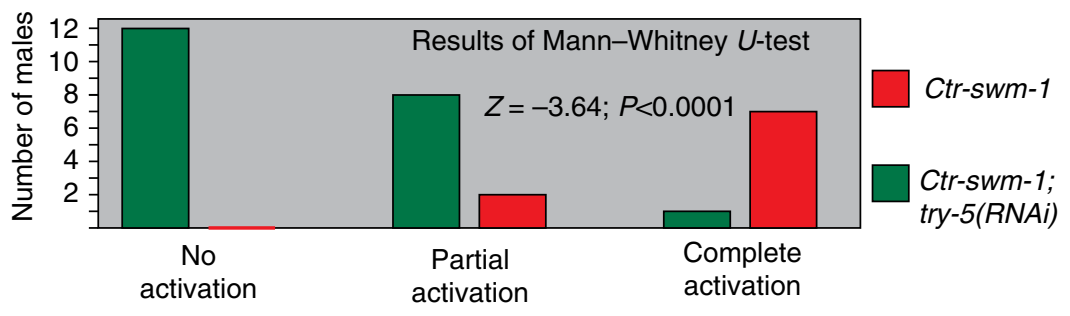

C

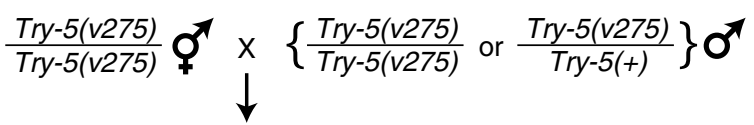

(1) Did the male sire children?

(2) What was the father's genotype?

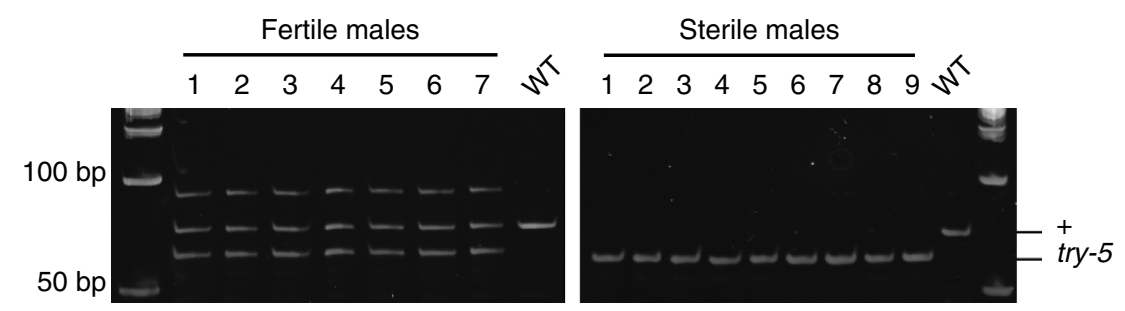

Figure 5 | C. tropicalis TRY-5 is required for male fertility. (a) Photomicrographs of adult males of the indicated genotypes, scored 2 days after the L4 larval stage. The ctr-swm-1(v294) mutation causes male sperm to activate before ejaculation, resulting in a characteristic rough appearance. This activation depends on TRY-5, because it is suppressed by knocking down try-5 with RNAi, (using $2.4 \mu \mathrm{g} \mu \mathrm{I}^{-1}$ double-stranded RNA). (b) Histogram showing the extent of sperm activation in C. tropicalis males that were either him-8; swm-1(v294) or him-8; swm-1(v294); try-5(RNAi). (c) Results of individual crosses between Ctr-try-5 mothers and single males that were either homozygous or heterozygous for try-5. After mating had occurred (as judged by the presence of a plug), the plates were scored for progeny and the $P_{0}$ males were removed for PCR analysis. All fertile males were heterozygotes and all sterile males were try-5 homozygotes. The upper band is a byproduct of the PCR reaction found in animals heterozygous for these two alleles.

a

b

Brood size of $C$. briggsae try-5 spe- 19 mothers

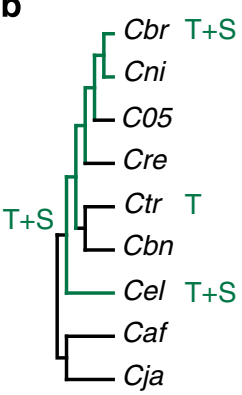

Figure 6 | The TRY-5 and SPE-8 pathways were redundant in ancestral males. (a) Analysis of crosses testing the fertility of Cbr-try-5(v141) males that were heterozygous or homozygous for spe-19(v166). We selected individual non-Unc male progeny from him- 8 ; try-5 + unc-51/ try-5 spe-19+ mothers. After each male was crossed with a try-5 spe-19 sterile mother, its genotype was determined by the PCR. Successful crosses were identified by mating plugs and scored for offspring the following day. Statistical calculations were done using vassarstats.net/utest.html. (b) Phylogeny of the elegans group, showing male sperm activation pathways ( $T=$ TRY -5 and $S=S P E-8$ group). On the basis of parsimony, ancestral males used both the TRY-5 and SPE- 8 pathways. 


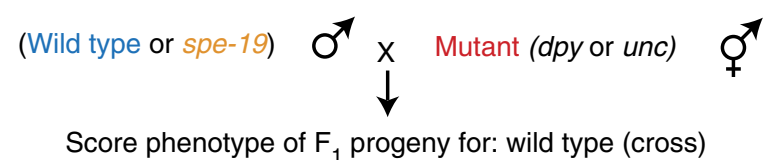

mutant (self)

b

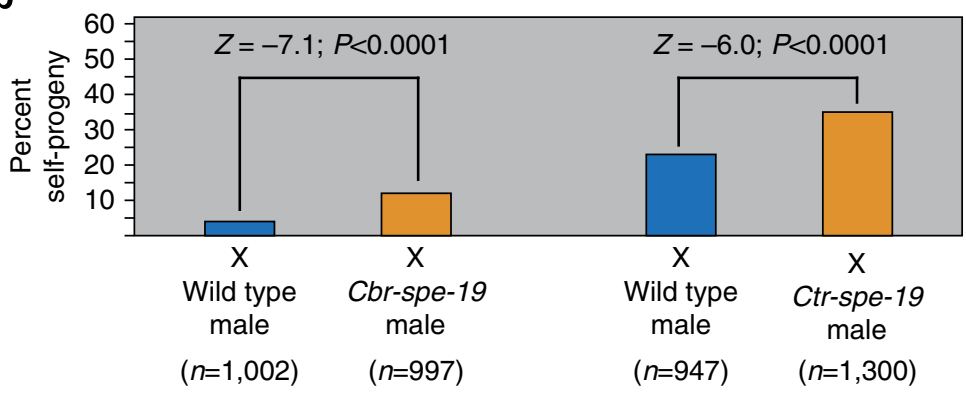

C

Ctr XX hermaphrodites $\quad$ Ctr XO males

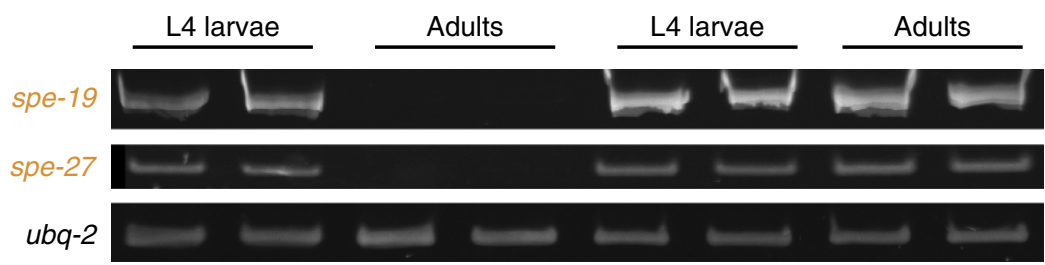

Figure 7 | The spe-19 gene helps produce optimal sperm in C. briggsae and C. tropicalis. (a) Method for measuring the ability of male sperm to compete with hermaphrodite sperm. After a brief mating, the progeny were counted for several days. To ensure that male-derived sperm were present during scoring, all data were from intermediate days, which were both preceded and followed by days with cross progeny. The mutations were cbr-spe-19(v166) or ctr-spe-19(v218) and the markers were cbr-dpy-11 or ctr-unc-23. (b) The significance of the difference between the pairs of proportions was calculated at vassarstats.net/propdiff_ind.html. (c) Semi-quantitative RT-PCR analysis of RNA from independent samples of each indicated age and sex reveals that $C$. tropicalis males express genes of the spe- 8 group. The ubiquitin gene $u b q-2$ was a loading control.

comparisons through gene editing. Although RNAi has been invaluable for evolutionary studies, phenotypes often vary between species because of differential sensitivity to $\mathrm{RNAi}^{37}$, rather than because the targeted pathways have changed. Even more problematic, some phenotypes (like those of nematode sperm) are often recalcitrant to RNAi. By contrast, gene editing using TALENs ${ }^{26-28}$ or CRISPRs ${ }^{38,39}$ can produce comparable alleles in each species. As these mutations allow rigorous comparisons of phenotype, we anticipate that their use will soon become routine.

\section{Methods}

Strains and genetics. C. briggsae mutants were derived from the wild isolate AF16 (ref. 40). They include: LGI: him-8(v188) (ref. 28); LGIV: she-1(v49) (ref. 41); LGV: $d p y-11(v 241)$ (ref. 28), unc-51(v205) (ref. 28) and new mutations described in the manuscript. C. nigoni RNAi experiments were done using JU1421 (M. Felix, pers. comm.). C. tropicalis mutants were derived from the wild isolate JU1373 (ref. 8). They include unc-23(v277) (ref. 28), him-8(v287), which deletes $9 \mathrm{nt}$ and adds $4 \mathrm{nt}$, and those described in the figures.

Cloning and sequencing. To identify the C. briggsae spe-12 gene, we used degenerate nucleotides designed according to the CODE-HOP protocol ${ }^{42}$. Missing portions of Ctr-spe-8 and C. sp. 5 spe-27 were amplified from genomic DNA using flanking primers. PCR products were purified with a PCR Purification kit (Qiagen) and sequenced (GeneWiz). Primers are listed in Supplementary Table 1.

RNA interference. Templates were amplified from mixed stage complementary DNA or genomic DNA by the PCR, with primers that contained a T7 promoter (Supplementary Table 1), purified with a PCR Purification kit (Qiagen) and transcribed using MegaScript (Ambion). After annealing, double-stranded RNA was purified with MegaClear (Ambion). RNAi was performed by injection ${ }^{43}$.
Semi-quantitative RT-PCR. Groups of five worms of the desired age and sex were collected and processed as described ${ }^{44}$; two independent samples were prepared to confirm reproducibility. RT-PCR was carried out using HotMaster Taq DNA polymerase (5PRIME) and MMLV Reverse Transcriptase (Invitrogen). PCR reactions were run for 35 cycles using primers from Supplementary Table 1.

Real-time quantitative RT-PCR. Groups of five worms of the desired age and sex were prepared as described above; at least three independent biological replicates and two technical replicates were assayed for each data point. For each reaction, $1 / 20$ of the total complementary DNA sample was used in a final volume of $25 \mu \mathrm{l}$, which included $12.5 \mu \mathrm{l}$ of FastStart Universal SYBR Green Master (Rox, Roche) and $6 \mu \mathrm{M}$ primers (Supplementary Table 1). Amplification was 40 cycles, using Applied Biosystems 7500 Real-Time PCR Systems. Samples that did not show detectable amplification by the final cycle were arbitrarily assigned a Ct value of 40

The $\Delta \mathrm{Ct}$ values for $s w m-1$ and try-5 were calculated for each group of L4 larvae, and normalised to control $u b q-2$ transcript levels. Afterwards, $-\Delta \Delta \mathrm{Ct}$ values were calculated for each group by comparing transcript levels for each sex and species with the corresponding swm-1 levels for those animals. Error bars are based on standard error of the mean.

Microscopy. Sperm were isolated as described ${ }^{9}$. Whole worms and isolated sperm were observed with differential interference contrast microscopy. Images were captured with a Zeiss Axiocam digital camera and Zeiss AxioVision software and assembled using Adobe Photoshop.

TALEN knockout mutants. TALENs were designed and produced as described ${ }^{28}$ (Supplementary Table 2). To create mutants, pairs of TALEN messenger RNAs were injected into the gonads of adult hermaphrodites ${ }^{26}$. At $20^{\circ} \mathrm{C}$, the $\mathrm{F}_{1}$ progeny from a 6 to $32 \mathrm{~h}$ time window were singled to new plates at the L4 stage, and those $\mathrm{F}_{1}$ animals that carried new mutations were identified by phenotype or by PCR analysis of the target site (primers in Supplementary Table 1). 


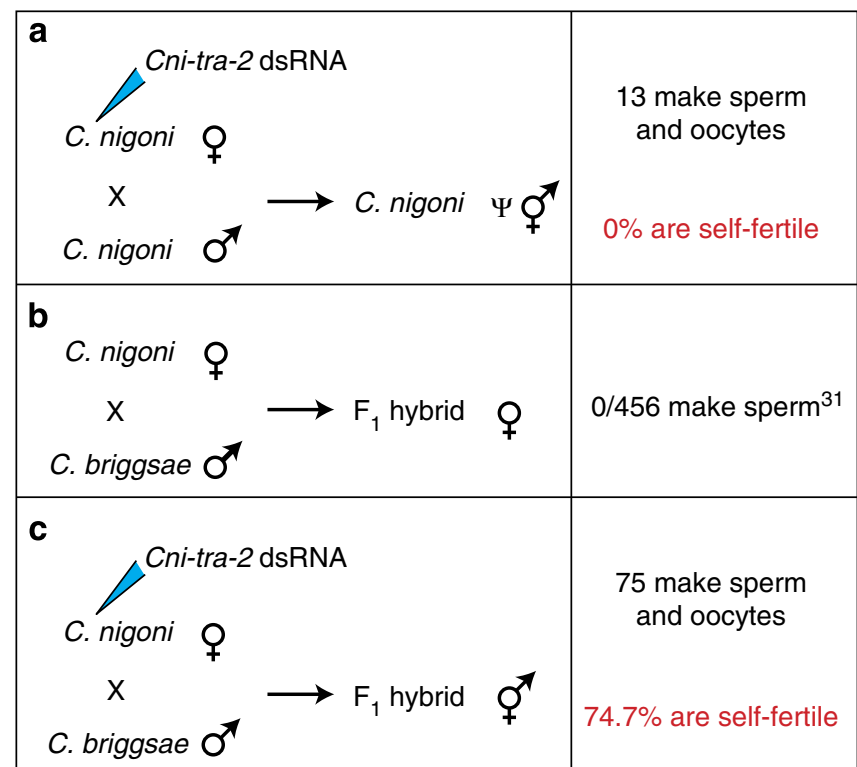

Figure 8 | C. briggsae contains a dominant gene that can activate sperm. (a) C. nigoni tra-2(RNAi) XX animals can develop as pseudohermaphrodites, just as those observed in C. remanei ${ }^{9}$. Out of $109 \mathrm{~F}_{1} X X$ progeny we observed 54 females, 13 pseudohermaphrodites and 42 Mog animals (which produced only sperm but had female bodies). The pseudohermaphrodites were not self-fertile and their sperm did not appear to have activated. (b) C. briggsae and C. nigoni are sister species that produce fertile female progeny; these hybrid animals do not make sperm ${ }^{31}$. (c) When tra-2 activity was knocked down by RNAi in the hybrid progeny (using $1 \mu g \mathrm{l}^{-1}$ double-stranded RNA), most of the animals that produced sperm were selffertile. Out of $119 F_{1} X X$ progeny we observed 56 self-fertile hermaphrodites but only 19 animals that laid oocytes and did not lay eggs, which implies that they produced inactive sperm. This result shows that $C$. briggsae contains a dominant factor that activates sperm, which is consistent with models in which C. briggsae hermaphrodites secrete an activating signal.

a

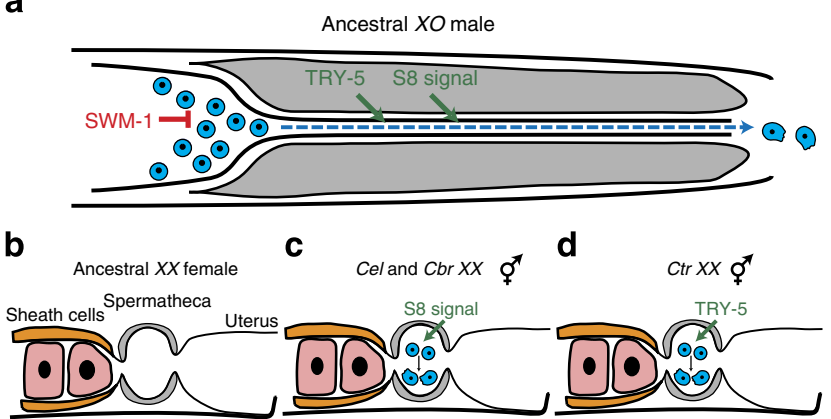

Figure 9 | The TRY-5 and SPE-8 pathways were redundant in ancestral males. (a) Model showing redundant sperm activation signals in ancestral males. These signals were produced in the somatic gonad and released in seminal fluid during ejaculation. S8 signal = unknown signal that acts on the SPE-8 group. (b-d) C. elegans and C. briggsae hermaphrodites co-opted the S8 signal to become self-fertile, but $C$. tropicalis hermaphrodites co-opted TRY-5.

Fertility assays. To assess hermaphrodite fertility, individual $\mathrm{L} 4$ animals were singled and transferred daily to new plates. After the mother was removed, each plate was scored by inspection for the number of progeny. Male fertility was assayed similarly, after crosses between single males and single females or hermaphrodites.

\section{References}

1. Darwin, C. On the Origin of Species by Means of Natural Selection, of the Preservation of Favored Races in the Struggle for Life (John Murray, 1859).

2. True, J. R. \& Carroll, S. B. Gene co-option in physiological and morphological evolution. Annu. Rev. Cell Dev. Biol. 18, 53-80 (2002).

3. Prud'homme, B. et al. Body plan innovation in treehoppers through the evolution of an extra wing-like appendage. Nature 473, 83-86 (2011).

4. Kijimoto, T., Pespeni, M., Beckers, O. \& Moczek, A. P. Beetle horns and horned beetles: emerging models in developmental evolution and ecology. Wiley Interdiscip. Rev. Dev. Biol. 2, 405-418 (2013).

5. Ellis, R. E. \& Lin, S. Y. The evolutionary origins and consequences of selffertility in nematodes. F1000Prime Rep. 6, 62 (2014).

6. Cho, S., Jin, S. W., Cohen, A. \& Ellis, R. E. A phylogeny of Caenorhabditis reveals frequent loss of introns during nematode evolution. Genome Res. 14, 1207-1220 (2004).

7. Kiontke, K. et al. Caenorhabditis phylogeny predicts convergence of hermaphroditism and extensive intron loss. Proc. Natl Acad. Sci. USA 101, 9003-9008 (2004).

8. Kiontke, K. C. et al. A phylogeny and molecular barcodes for Caenorhabditis, with numerous new species from rotting fruits. BMC Evol. Biol. 11, 339 (2011).

9. Baldi, C., Cho, S. \& Ellis, R. E. Mutations in two independent pathways are sufficient to create hermaphroditic nematodes. Science 326, 1002-1005 (2009).

10. L'Hernault, S. W., Shakes, D. C. \& Ward, S. Developmental genetics of chromosome I spermatogenesis-defective mutants in the nematode Caenorhabditis elegans. Genetics 120, 435-452 (1988).

11. Muhlrad, P. J., Clark, J. N., Nasri, U., Sullivan, N. G. \& LaMunyon, C. W. SPE8 , a protein-tyrosine kinase, localizes to the spermatid cell membrane through interaction with other members of the SPE- 8 group spermatid activation signaling pathway in C. elegans. BMC Genet. 15, 83 (2014).

12. Nance, J., Minniti, A. N., Sadler, C. \& Ward, S. spe-12 encodes a sperm cell surface protein that promotes spermiogenesis in Caenorhabditis elegans. Genetics 152, 209-220 (1999).

13. Geldziler, B., Chatterjee, I. \& Singson, A. The genetic and molecular analysis of spe-19, a gene required for sperm activation in Caenorhabditis elegans. Dev. Biol. 283, 424-436 (2005).

14. Minniti, A. N., Sadler, C. \& Ward, S. Genetic and molecular analysis of spe-27, a gene required for spermiogenesis in Caenorhabditis elegans hermaphrodites. Genetics 143, 213-223 (1996).

15. Nance, J., Davis, E. B. \& Ward, S. spe-29 encodes a small predicted membrane protein required for the initiation of sperm activation in Caenorhabditis elegans. Genetics 156, 1623-1633 (2000).

16. Ellis, R. E. \& Stanfield, G. M. The regulation of spermatogenesis and sperm function in nematodes. Semin. Cell Dev. Biol. 29C, 17-30 (2014).

17. Liu, Z., Chen, L., Shang, Y., Huang, P. \& Miao, L. The micronutrient element zinc modulates sperm activation through the SPE-8 pathway in Caenorhabditis elegans. Development 140, 2103-2107 (2013).

18. Smith, J. R. \& Stanfield, G. M. TRY-5 Is a sperm-activating protease in Caenorhabditis elegans seminal fluid. PLoS Genet. 7, e1002375 (2011).

19. Zhao, Y. et al. Nematode sperm maturation triggered by protease involves sperm-secreted serine protease inhibitor (Serpin). Proc. Natl Acad. Sci. USA 109, 1542-1547 (2012).

20. Stanfield, G. M. \& Villeneuve, A. M. Regulation of sperm activation by SWM-1 is required for reproductive success of C. elegans males. Curr. Biol. 16, 252-263 (2006).

21. Fenker, K. E. et al. SLC6 family transporter SNF-10 is required for proteasemediated activation of sperm motility in C. elegans. Dev. Biol. 393, 171-182 (2014).

22. Muhlrad, P. J. \& Ward, S. Spermiogenesis initiation in Caenorhabditis elegans involves a casein kinase 1 encoded by the spe-6 gene. Genetics 161, 143-155 (2002).

23. Gosney, R., Liau, W. S. \& LaMunyon, C. W. A novel function for the presenilin family member spe-4: inhibition of spermatid activation in Caenorhabditis elegans. BMC Dev. Biol. 8, 44 (2008).

24. Liau, W. S., Nasri, U., Elmatari, D., Rothman, J. \& LaMunyon, C. W. Premature sperm activation and defective spermatogenesis caused by loss of spe-46 function in Caenorhabditis elegans. PLoS ONE 8, e57266 (2013).

25. Hill, K. L. \& L'Hernault, S. W. Analyses of reproductive interactions that occur after heterospecific matings within the genus Caenorhabditis. Dev. Biol. 232, 105-114 (2001).

26. Wood, A. J. et al. Targeted genome editing across species using ZFNs and TALENs. Science 333, 307 (2011).

27. Lo, T. W. et al. Using TALENs and CRISPR/Cas9 to engineer insertions and deletions. Genetics 195, 331-348 (2013).

28. Wei, Q., Shen, Y., Chen, X., Shifman, Y. \& Ellis, R. E. Rapid creation of forward-genetics tools for C. briggsae using TALENs: lessons for nonmodel organisms. Mol. Biol. Evol. 31, 468-473 (2014).

29. Baldi, C., Viviano, J. \& Ellis, R. E. A bias caused by ectopic development produces sexually dimorphic sperm in nematodes. Curr. Biol. 21, 1416-1420 (2011). 
30. Félix, M. A., Braendle, C. \& Cutter, A. D. A streamlined system for species diagnosis in Caenorhabditis (Nematoda: Rhabditidae) with name designations for 15 distinct biological species. PLoS ONE 9, e94723 (2014).

31. Woodruff, G. C., Eke, O., Baird, S. E., Félix, M. A. \& Haag, E. S. Insights into species divergence and the evolution of hermaphroditism from fertile interspecies hybrids of Caenorhabditis nematodes. Genetics 186, 997-1012 (2010).

32. Shubin, N., Tabin, C. \& Carroll, S. Deep homology and the origins of evolutionary novelty. Nature 457, 818-823 (2009).

33. Chipman, A. D. Parallel evolution of segmentation by co-option of ancestral gene regulatory networks. Bioessays 32, 60-70 (2010).

34. Muller, G. B. Evo-devo: extending the evolutionary synthesis. Nat. Rev. Genet. 8, 943-949 (2007).

35. Losos, J. B. Convergence, adaptation, and constraint. Evolution 65, 1827-1840 (2011).

36. Stern, D. L. The genetic causes of convergent evolution. Nat. Rev. Genet. 14, 751-764 (2013).

37. Nuez, I. \& Félix, M. A. Evolution of Susceptibility to Ingested Double-Stranded RNAs in Caenorhabditis Nematodes. PLoS ONE 7, e29811 (2012).

38. Gilbert, L. A. et al. CRISPR-mediated modular RNA-guided regulation of transcription in eukaryotes. Cell 154, 442-451 (2013).

39. Dickinson, D. J., Ward, J. D., Reiner, D. J. \& Goldstein, B. Engineering the Caenorhabditis elegans genome using Cas9-triggered homologous recombination. Nat. Methods 10, 1028-1034 (2013).

40. Fodor, A., Riddle, D. L., Nelson, F. K. \& Golden, J. W. Comparison of a new wild-type Caenorhabditis briggsae with laboratory strains of C. briggsae and C. elegans. Nematologica 29, 203-217 (1983).

41. Guo, Y., Lang, S. \& Ellis, R. E. Independent recruitment of F box genes to regulate hermaphrodite development during nematode evolution. Curr. Biol. 19, 1853-1860 (2009).

42. Rose, T. M., Henikoff, J. G. \& Henikoff, S. CODEHOP (COnsensus-DEgenerate Hybrid Oligonucleotide Primer) PCR primer design. Nucleic Acids Res. 31, 3763-3766 (2003)

43. Fire, A. et al. Potent and specific genetic interference by double-stranded RNA in Caenorhabditis elegans. Nature 391, 806-811 (1998).

44. Chen, P. J. \& Ellis, R. E. TRA-1A regulates transcription of fog-3, which controls germ cell fate in C. elegans. Development 127, 3119-3129 (2000).
45. Edgar, R. C. MUSCLE: multiple sequence alignment with high accuracy and high throughput. Nucleic Acids Res. 32, 1792-1797 (2004).

46. Cserzo, M., Eisenhaber, F., Eisenhaber, B. \& Simon, I. On filtering false positive transmembrane protein predictions. Protein Eng. 15, 745-752 (2002).

47. Baird, S. E., Sutherlin, M. E. \& Emmons, S. W. Reproductive isolation in Rhabditidae (Nematoda: Secernentea); mechanisms that isolate six species of three genera. Evolution 46, 585-594 (1992).

\section{Acknowledgements}

We thank the National Science Foundation for support (IOS 1021128) and David Fitch Karin Kiontke and Marie-Anne Felix for strains. Additional strains were provided by the CGC, which is funded by NIH Office of Research Infrastructure Programs (P40 OD010440).

\section{Author contributions}

R.E.E. conceived the project, directed it and carried out the computational analyses. Q.W. performed the majority of the experiments, with the assistance of Y.Z. in the production of Ctr-swm-1 mutants, Y.G. in the characterisation of pseudohermaphrodites, J.S. in determining the brood size for mutant strains and R.S. in the identification of some genes. The manuscript was written by R.E.E. and Q.W

\section{Additional information}

Accession codes: The C. briggsae spe-12 sequence has been deposited in the GenBank database with accession code KP123338.

Supplementary Information accompanies this paper at http://www.nature.com/ naturecommunications

Competing financial interests: The authors declare no competing financial interests.

Reprints and permission information is available online at http://npg.nature.com/ reprintsandpermissions/

How to cite this article: Wei, Q. et al. Co-option of alternate sperm activation programs in the evolution of self-fertile nematodes. Nat. Commun. 5:5888 doi: $10.1038 /$ ncomms6888 (2014) 